\title{
Prediction of Hole Expansion Ratio of Titanium Alloys Using $\mathbf{R}$ Programming
}

\author{
J. S. Kwame ${ }^{1}$, E. Yakushina ${ }^{2}$, P. Blackwell ${ }^{1,2}$ \\ 1. Department of Design, Manufacturing and Engineering Management, University of Strathclyde, United \\ Kingdom \\ 2. Advanced Forming Research Centre (AFRC), University of Strathclyde, United Kingdom \\ E-mail:james.kwame@strath.ac.uk
}

Received: 9 September 2020; Accepted: 8 October 2020; Available online: 25 October 2020

\begin{abstract}
The hole expansion ratio (HER) is an important material property which defines the extent of edge formability of sheet metals. The stress states observed at the hole edge after the hole expansion test are similar to those seen during traditional uniaxial tensile deformation. This observation has provoked research, directed at ascertaining a correlation between the HER and tensile properties. In order to account for the forming behaviour of complex materials like titanium, a highly robust model that takes into account the material formability in all sheet-processing directions must be considered. The R programming language was used in this research to build a model fitting expression capable of predicting the HER as well as generating a regression model equation for titanium alloys, based on their thickness and Erichsen index number. The proposed regression model equation for predicting HER of titanium alloys exhibited an excellent statistical significance $(p=0.00076)$, indicating the robustness of the model fitting expression to predict HER values of titanium alloys. An accompanying adjusted $\mathrm{R}$ squared value of 0.9987 for the generated regression model equation also shows how well the regression line fits the data for accurate prediction of the HER of titanium alloys. A numerical validation analysis of the strength of the relationship derived between the predicted and the experimental HERs gave a correlation coefficient of 0.9884. This result shows a strong linear relationship between the experimental and predicted HER values of the titanium alloys with an average absolute error of $8.8 \%$.
\end{abstract}

Keywords: Titanium alloys; R programming; Hole expansion ratio; Erichsen index number; Abrasive water jet; Sheet thickness.

\section{Introduction}

The manufacture of components for aerospace application sometimes involves the integration of hole flanging parts with other segments. During forming operations, the pre-fabricated holes are often exposed to different processes including stretching, bending or even wrinkling. Sheet metal edge formability is a material property which defines the ability of a sheet metal to oppose edge failure during edge forming processes. The hole expansion test (HET) is the standard experimental testing technique used to quantify the edge formability of sheet metals according to ISO 16630:2017. The hole expansion ratio (HER) defines the extent of edge formability of a sheet material. The higher the HER, the higher the edge formability of materials. For a given sheet metal with an initial fabricated hole diameter $D_{0}$ prior to HET, and a final hole diameter $D_{f}$ at fracture after HET, the HER can be expressed as;

$$
H E R=\frac{D_{f}-D_{0}}{D_{0}} \times 100 \%
$$

Different sheet preparation methods are used to prepare sheet metal samples for HET. These machining methods are known to introduce defects onto the cut surfaces, which influences the edge formability of materials [1]. Zhang et al., [2] in their work observed that, burr up or down conditions in dual phase steel sheets influences the failure mode during HET. Some researchers have explored different damage criteria and finite element modelling (FEM) techniques to predict HER. Butcher et al., [3] in their work utilised FEM and Gurson-Tvergaard-Needleman (GTN) damage criteria to predict the HER of dual phase steel. Park et al., [4] utilised FEM based on different fracture criteria and a representative volume element, which accounts for microstructural effects to predict the HER of advanced high strength steels. Chung et al., [5] in their work developed a triaxiality dependent fracture criterion with hardening behaviour and stiffness deterioration within an anisotropic criterion to predict the HER and edge 
condition sensitivity of advanced high strength steel sheets. According to their research, these criteria were adopted within an FEM package to predict the HER.

Generally, the stress state observed at the hole edge tip after HET is the uniaxial stress state [6], which is similar to the stress state observed during a traditional uniaxial tensile test. This observation has prompted several researchers to correlate the HER to material tensile properties. Kumar De et al., [7] in their work on hot-rolled high-strength steels suggested that where strengthening mechanism of steels are the same, the ultimate tensile strength correlates well with the HER. Chen et al., [8] in their work on ultra-high strength steels observed that there is a strong inverse correlation between the HER and tensile strength with values below 640MPa and no change in HER for tensile strength above 840MPa. Fang et al., [9] in their study on C-Mn steels showed that higher values of the yield to tensile ratio correlates well with the HER. Adamczyk and Michal [10] utilised a multiple linear regression model for correlating the HER to the transverse total elongation and average plastic anisotropy of high-strength cold-rolled steels. Some works have also recognised the difficulty in correlating material tensile properties to the HER. Kim et al., [11] in their work using basic tensile properties to predict the HER of thin steel sheets with a machined hole recognised the difficulty in correlating the parameters. Their work proposed evaluating the width strains from the measured normal anisotropy as well as considering the tensile elongation only along the sheet rolling direction. They also suggested that utilising the width strain in uniaxial tension to represent the planar anisotropy would improve the correlation with the HER.

Generally, initial assessment of the formability of sheet metals is mostly based on the total elongation values determined from tensile deformation. However, this approach may be insufficient and unreliable when accounting for sheet forming behaviour. Tension tests are done under uniaxial conditions whiles HETs are conducted under different loading states depending on the tool geometry [12]. Moreover, the above-mentioned studies were mostly conducted on steels, which have relatively less complex textures compared to titanium and its alloy. Singh and Schwarzer [13] in their work on texture and anisotropy of titanium and its alloys showed that texture evolution is a function of several characteristics such as deformation mode, deformation temperature, original texture and microstructure, deformation rate as well as constituent elements. Considering the highly anisotropic nature of titanium and its alloys, the feasibility of selecting a preferred material property direction for correlation with the HER is complex. An attempt to characterise titanium materials in several sheet directions in a bid to identify a preferred orientation could be cost intensive in terms of material, equipment time and operator time. Therefore, for a more robust approach, there is the need to consider a material property that caters for the holistic response of materials in terms of their ductility and formability in all sheet-processing directions. The Erichsen index number (EI) derived from the Erichsen cupping test is utilised in this research. The Erichsen cupping test has become an important technique for evaluating the formability of sheet metals. The plastic deformation via biaxial stretching of a fully constrained sheet metal also makes the technique suitable for ascertaining the ductile behaviour of sheet metals. The usefulness of this technique has drawn interest in its suitability for the assessment of sheet metals for stamping and deep drawing operations. The Erichsen index number is a material parameter derived from the punch displacement until fracture during the Erichsen cupping test. Some researchers have adopted optical non-contact measurement methods to characterise the Erichsen index number. Kocańda and Jasiński [14] in their work have shown that laser speckles can be utilised in evaluating the cupping height during localised necking as well as before fracture occurs during Erichsen testing. Aydin et al., [15] in their work also utilised a digital image correlation method to evaluate the fracture cup height of Ck75 steels during a cupping test.

The HET and Erichsen cupping test are adequate techniques for the evaluation of sheet metal stretchflangeability, formability and ductility mainly because, they prevent draw-in of the sheet during the testing process [16]. A regression model expression is proposed in this research to forecast the HER of titanium alloys by taking into account the sheet thickness and the $E I$ of the materials. A thickness parameter was introduced into the proposed predictive regression model expression due to their reported impact on part formability. Dilmec et al., [17] in their work on the influence of sheet thickness and anisotropy on the forming limit curve (FLC) of AA 2024-T4 aluminium alloys revealed that an increase in the sheet thickness increases the FLC level. Zadpoor et al., [18] in their work also investigated the impact of sheet thickness on the formability of AA 2024-T3 and AA7075-T6 aluminium alloys during tensile testing and air bending. Their work reported that the minimum bending radius increases with increasing sheet thickness. For the tensile test, strains at optimum stress and ultimate strain were observed to increase with increasing sheet thickness up to $2 \mathrm{~mm}$. Hashemi et al., [19] in their work examined the influence of material properties and initial sheet thickness on the formability of pure copper and st14 steel by utilising hydro-mechanical deep drawing assisted by radial pressure. Their work found that for reducing sheet thickness in both materials, the thickness reduction percentage in critical areas decreases.

A model fitting expression developed in R programming, capable of predicting the HER as well as generating a constitutive regression model equation for titanium alloys, based on their Erichsen index number $(E I)$ and material thickness $(t)$, is proposed in this research. 


\section{Experimental procedure}

\subsection{The materials}

Different titanium alloy sheets with varied thicknesses were used in this work. The materials with their corresponding chemical compositions are shown in Table 1 . In order to capture the full range of material property response in the predictive regression model, a commercially pure titanium alloy (Ti-35A) and one with high strength (Ti-64) were included. Ti-35A has the highest purity of the four pure titanium grades and has the lowest strength with high room temperature formability and ductility. Ti-64 is the most utilised titanium alloy and represents about $60 \%$ of the entire titanium production, with the commercially pure alloys accounting for $20 \%$ and all other alloy forms of titanium constituting the remaining $20 \%$ of total titanium production [20].

Table 1. Chemical composition of titanium alloys in Wt.\%

\begin{tabular}{ccccccccc}
\hline Material & $\boldsymbol{A l}$ & $\boldsymbol{V}$ & $\boldsymbol{F e}$ & $\boldsymbol{O}$ & $\boldsymbol{C}$ & $\boldsymbol{N}$ & $\boldsymbol{H}$ & Residual \\
\hline Ti-6Al-4V & $5.5-6.8$ & $3.5-4.5$ & 0.3 & 0.2 & 0.08 & 0.05 & 0.015 & 0.4 \\
Ti-2Al-1.5V & $1.5-2.5$ & $1.0-2.0$ & 0.2 & 0.18 & 0.03 & 0.03 & 0.015 & 0.4 \\
CP-Ti (Grade 2) & 0 & 0 & 0.3 & 0.25 & 0.1 & 0.03 & 0.015 & 0.4 \\
Ti-3Al-2.5V & $2.5-3.5$ & $2.0-3.0$ & 0.25 & 0.12 & 0.05 & 0.02 & 0.015 & 0.4 \\
Ti-35A & 0 & 0 & 0.2 & 0.18 & 0.08 & 0.03 & 0015 & 0.4 \\
\hline
\end{tabular}

\subsection{Hole expansion test and Erichsen cupping test}

The hole expansion test was performed on a Zwick/ Roell BUP 1000 machine with the test parameters controlled by the Zwick testXpert software. Abrasive water jet (AWJ) machining was used to fabricate circular disc test samples with an outer diameter of $200 \mathrm{~mm}$ and an inner hole diameter of $20 \mathrm{~mm}$. A Nakajima punch with a diameter of $100 \mathrm{~mm}$ was used to deform the samples at a speed of $1 \mathrm{~mm} / \mathrm{s}$ until a crack at the hole edge was observed. A blankholder force of $150 \mathrm{kN}$ was used to ensure that the sheet is prevented from drawing-in during the deformation process. A schematic setup of the HET is shown in Figure 1. Three replicates of the AWJ machined hole edges were tested for each titanium alloy. The HER was evaluated for each deformed titanium alloy according to equation (1).

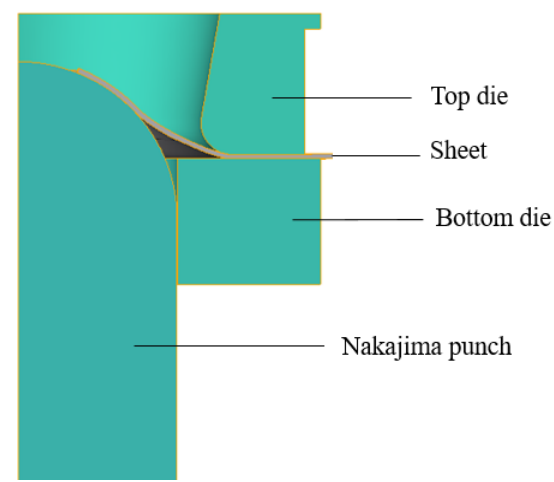

Figure 1. Schematic of the hole expansion test

An Erichsen cupping test with a tool set consistent with ISO 20482:2013, was conducted on the titanium alloys. A punch with a $20 \mathrm{~mm}$ spherical head was used to deform blanks with a dimension of $75 \mathrm{~mm} \times 75 \mathrm{~mm}$, until fracture occurred. Grease was used as a lubricant to minimise friction between the punch and the sheet metal sample. The extent of formability was assessed by measuring the distance from the initial punch contact to the point of fracture. This height represents the Erichsen index number. Three replicates of the Erichsen cupping test was conducted for each titanium grade. A random speckle pattern consisting of black dots on a white paint background were utilised for mapping the surface strains with a Gom Aramis optical non-contact measurement system. This tracks the fullfield displacement during the deformation process.

\subsection{Model description and development}

The $R$ programming language is an open-source execution of the $\mathrm{S}$ functional programming language. $R$ is an object oriented functional programming language, convenient for high-level scientific programming, data analysis as well as modelling. For computationally cumbersome tasks, languages like C, $\mathrm{C}++$, Python and Fortran could be linked and called up as required [21, 22, 23].

For this research, $R$ scripts were coded and implemented in $R$ studio programming language to predict the HER of titanium alloys based on their $E I$ and $t$ values. The underlining model fitting expression executed in this work 
to predict HER of titanium alloys as well as generate regression model equation is given by;

$$
\operatorname{lm}(\log (\text { HER }) \sim \text { Erichsen Index + poly (thickness, 2), data = experimental data) }
$$

A logarithmic transform and polynomial functions were employed in the proposed regression model expression in order to capture the non-linearity in the data fitting process. Figure 2 shows the flowchart of the sequence logic followed to execute the model fitting expression governed by the $R$ codes, for the accurate prediction of HER values for titanium alloys. An excel sheet location containing the experimental data with HER, EI and $t$ values were loaded into the $R$-studio software, guided by the $R$ codes. The model fitting expression according to equation (2), was then run to calibrate the resulting regression model. Based on the obtained $p$-value and the adjusted $R$ squared $\left(R_{A d j}^{2}\right)$ values, the generated regression model equation could be accepted or rejected. A statistically significant model (overall $p<0.05$ ) with high correlation $\left(R_{A d j}^{2}\right.$ close to 1$)$ translates into a very robust regression model equation capable of predicting the HER values. Depending on the statistical strength of the generated regression model equation, the HER of an unknown titanium alloy can now be predicted based on known EI and $t$ input values.

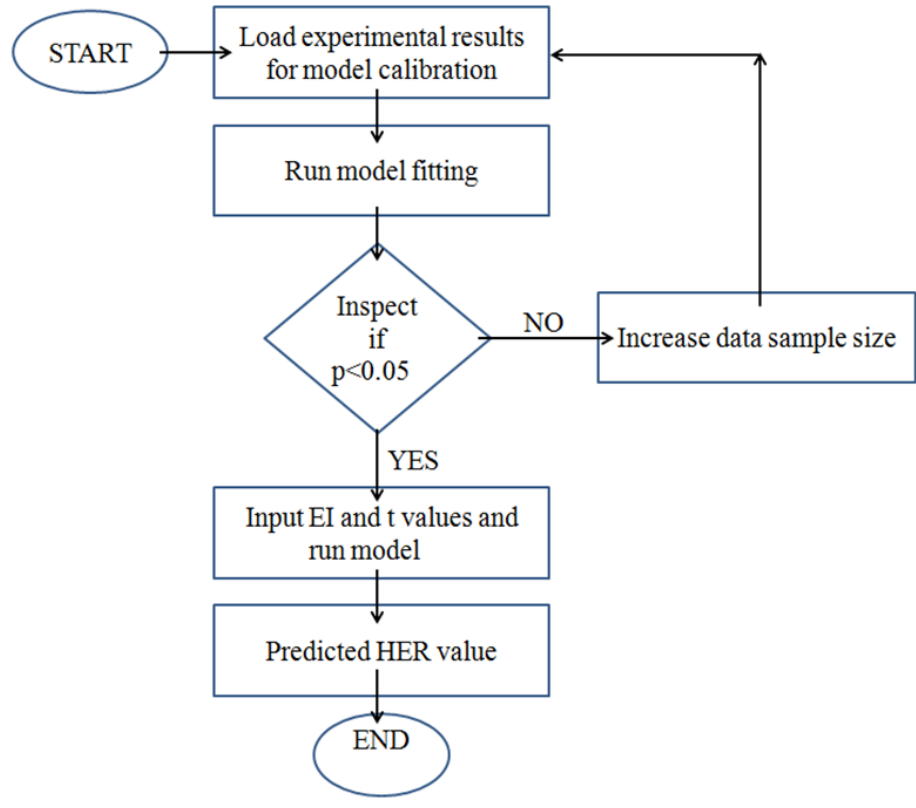

Figure 2. HER prediction flowchart

\subsection{Model performance assessment criteria}

A significant test, based on the enumeration of the $p$-value is used in this work. The $p$-value helps to ascertain if differences seen between the test results and the null hypothesis are because of the null hypothesis failure, or due to sampling variation. Generally, low $p$-values depict that there is a low probability of the null hypothesis to produce higher values than those obtained. The levels of significance for different limits of $p$-values are shown in Table 2. Statistical significance level was considered at $p<0.05$ in this work.

Table 2. p-value span for levels of significance [22]

\begin{tabular}{cc}
\hline p-value Span & Description \\
\hline $0-0.001$ & Extremely significant \\
$0.001-0.01$ & Highly significant \\
$0.01-0.05$ & Statistically significant \\
$0.05-0.1$ & Could be significant \\
$0.1-1$ & Not significant \\
\hline
\end{tabular}

For the model to be robust, it should be able to capture variations observed in the response variable by adopting some predictor variables. The total variation in the response variable, the $y$ values, about a mean point is defined by the total sum of squares, SST [22];

$$
S S T=\sum\left(y_{i}-\bar{y}\right)^{2}
$$


Equation (3) can be expressed algebraically as;

$$
\sum\left(y_{i}-\bar{y}\right)^{2}=\sum\left(y_{i}-\hat{y}_{i}\right)^{2}+\sum\left(\hat{y}_{i}-\bar{y}\right)^{2}
$$

where, $\sum\left(y_{i}-\hat{y}_{i}\right)^{2}$ is the residual sum of squares (RSS) and $\sum\left(\hat{y}_{i}-\bar{y}\right)^{2}$ is the regression sum of squares (SSReg), defined as the total variation in the fitted model about the mean.

The SST, RSS and SSReg thus enables for a proper definition of an appropriate regression line that fits the data. In order to ascertain how well the regression line fits the given data, the coefficient of determination $R^{2}$ was adopted;

$$
R^{2}=1-\frac{R S S}{S S T}=1-\frac{\sum\left(y_{i}-\hat{y}_{i}\right)^{2}}{\sum\left(y_{i}-\bar{y}_{i}\right)^{2}}
$$

Putting SST in equation (3) back into equation (5)

$$
R^{2}=\frac{\sum\left(y_{i}-\bar{y}\right)^{2}-\sum\left(y_{i}-\hat{y}_{i}\right)^{2}}{\sum\left(y_{i}-\bar{y}\right)^{2}}
$$

But, from equation (4); $\sum\left(y_{i}-\bar{y}\right)^{2}=\sum\left(y_{i}-\hat{y}_{i}\right)^{2}+\sum\left(\hat{y}_{i}-\bar{y}\right)^{2}$

Substituting equation (4) into equation (6);

$$
\begin{aligned}
R^{2} & =\frac{\sum\left(y_{i}-\hat{y}_{i}\right)^{2}+\sum\left(\hat{y}_{i}-\bar{y}\right)^{2}-\sum\left(y_{i}-\hat{y}_{i}\right)^{2}}{\sum\left(y_{i}-\bar{y}\right)^{2}} \\
R^{2} & =\frac{\sum\left(\hat{y}_{i}-\bar{y}\right)^{2}}{\sum\left(y_{i}-\bar{y}\right)^{2}}
\end{aligned}
$$

Equation (7) can be simplified as;

$$
R^{2}=\frac{\text { SSReg }}{\text { SST }}
$$

Thus, for values ranging between $0-1$, the regression line captures most of the variations in the data when $R^{2}$ is close to one.

Generally, $R^{2}$ represents the sample approximate of the proportion of variance, which are captured in the outcome variables, and are favoured upwards with respect to the explained population proportion of variance. In order to attenuate the limitations of $R^{2}$ and discourage the use of more predictor variables to get higher $R^{2}$ values, the adjusted $R^{2},\left(R^{2}\right.$ Adj $)$, are adopted to guard against spurious relationships. Thus, the $R^{2}$ Adj only increases when factors are highly correlated, for situations where more independent variables are added to the model. The sum of squares is divided by the degrees of freedom to account for the $R^{2}$ Adj. Generally, when considering simple regression models, $n-2$ are utilised for RSS and $n-1$ for SST [22].

For a given number of predictor variable $k$ and sample size $n$, the $R^{2}{ }_{\text {Adj }}$ can be expressed as [24];

$$
R_{A d j}^{2}=1-\left[\left(1-R^{2}\right)\left(\frac{n-1}{n-k-1}\right)\right]
$$

Putting equation (5) into equation (9), $R^{2}$ Adj can also be written as;

$$
R_{A d j}^{2}=1-\left[\frac{R S S}{S S T} \times\left(\frac{n-1}{n-k-1}\right)\right]
$$

The adjusted $R^{2}$ was used in this work to assess how well the regression line fits the data for accurate prediction of the HER of titanium alloys.

\section{Results and discussions}

\subsection{Formability of titanium alloys}

Table 3 shows the HER and the EI values of the studied titanium grades after the experimental trials. The varied formability performance of the titanium grades suggests that apart from the influence of machining induced edge 
surface defects on the HER, the material microstructures played an important role in the edge formability of the titanium alloys. The EI values are representative of the material ductility in all sheet-processing directions and reveals the extent of the material formability dependence on microstructure at room temperature. The unalloyed titanium sheets (Ti-35A and CP-Ti) showed high edge formability compared to their alloyed counterparts due to their superior room temperature ductility and lower strength. Ti-64 showed the lowest formability compared to the other titanium grades due to its high strength at room temperature. Similar limited room temperature formability as a result of low material hardening was recorded in Ti-64 during stretch forming [25].

Table 3. Formability at room temperature

\begin{tabular}{cccc}
\hline Material & $\boldsymbol{t}, \mathbf{~ m m}$ & $\boldsymbol{E I , ~} \mathbf{~ m m}$ & HER, \% \\
\hline Ti-3Al-2.5V & 1.60 & 5.889 & 30.096 \\
Ti-3Al-2.5V & 1.25 & 6.218 & 28.357 \\
Ti-2Al-1.5V & 1.27 & 8.980 & 52.529 \\
Ti-6Al-4V & 1.60 & 4.014 & 18.344 \\
CP-Ti (Grade 2) & 1.60 & 10.289 & 81.870 \\
Ti-35A & 1.00 & 11.313 & 157.317 \\
\hline
\end{tabular}

A graphical comparison of the performance trends observed in the material formability behaviour after HET and Erichsen cupping test is shown in Figure 3. The materials exhibited similar trends of formability response for both experimental techniques, thus showing the possibility of correlating test results from both techniques.

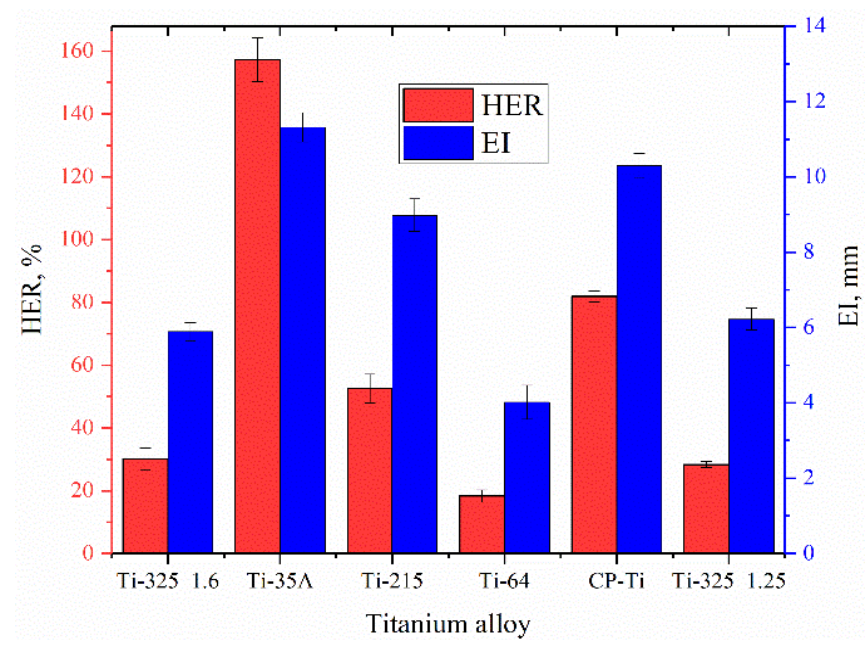

Figure 3. HER and EI assessment of titanium alloys

\subsection{Forming strains during Erichsen cupping test}

Figure 4 shows the forming strain evolution at the onset of localised necking prior to fracture during the testing process. Overall, high strain concentration sites were observed at the summit of the dome region indicating minimal friction between the spherical punch head and the test samples. Failure in Ti-64 was sudden with limited strain localisation (15.9\%) and no distinct necking prior to failure, Figure $4 c$. Similar trends were recorded in Ti64 after stretch forming with a plane strain forming limit minimum under 10\% [25]. In the present experiments $\mathrm{Ti}-35 \mathrm{~A}$ and CP-Ti pure grades showed the highest resistance to fracture (76.26\% and 64.46\% respectively, Figure $4 a, b)$, mainly due to their superior room temperature formability. Localised necking prior to fracture was observed in CP-Ti and Ti-35A, resulting in delayed fracture. The response of CP-Ti and Ti-64 to stretch forming are consistent with those observed in other works [26].

\subsection{Thinning evolution during Erichsen cupping test}

Figure 5 shows the material thinning evolution along the chosen section lengths of interest, at localised necking prior to fracture. Generally, optimum sheet thinning was observed around the dome summit prior to fracture during the biaxial stretching process. Reduced cupping height observed in Ti-64 reflected in their poor thinning tendencies (21.6\%) prior to failure, Figure $5 c$. The limited thinning noted in $\mathrm{Ti} 64$ is evident of the abrupt fracture of the part observed during the forming process. Ti-35A and CP-Ti pure grades exhibited the highest cupping height with an accompanying high formability, which reflected in their high resistance to thinning (64\% and 60.3\% respectively) prior to fracture, Figure $5 a, b$. Overall, the thinning tendencies exhibited by the titanium alloys are consistent with the forming performance observed after HET. The higher the thinning capability, the higher the formability of the 
material. In conventional sheet metal forming processes like incremental sheet forming, the maximum allowable thinning is an indicator of sheet metal formability [27].

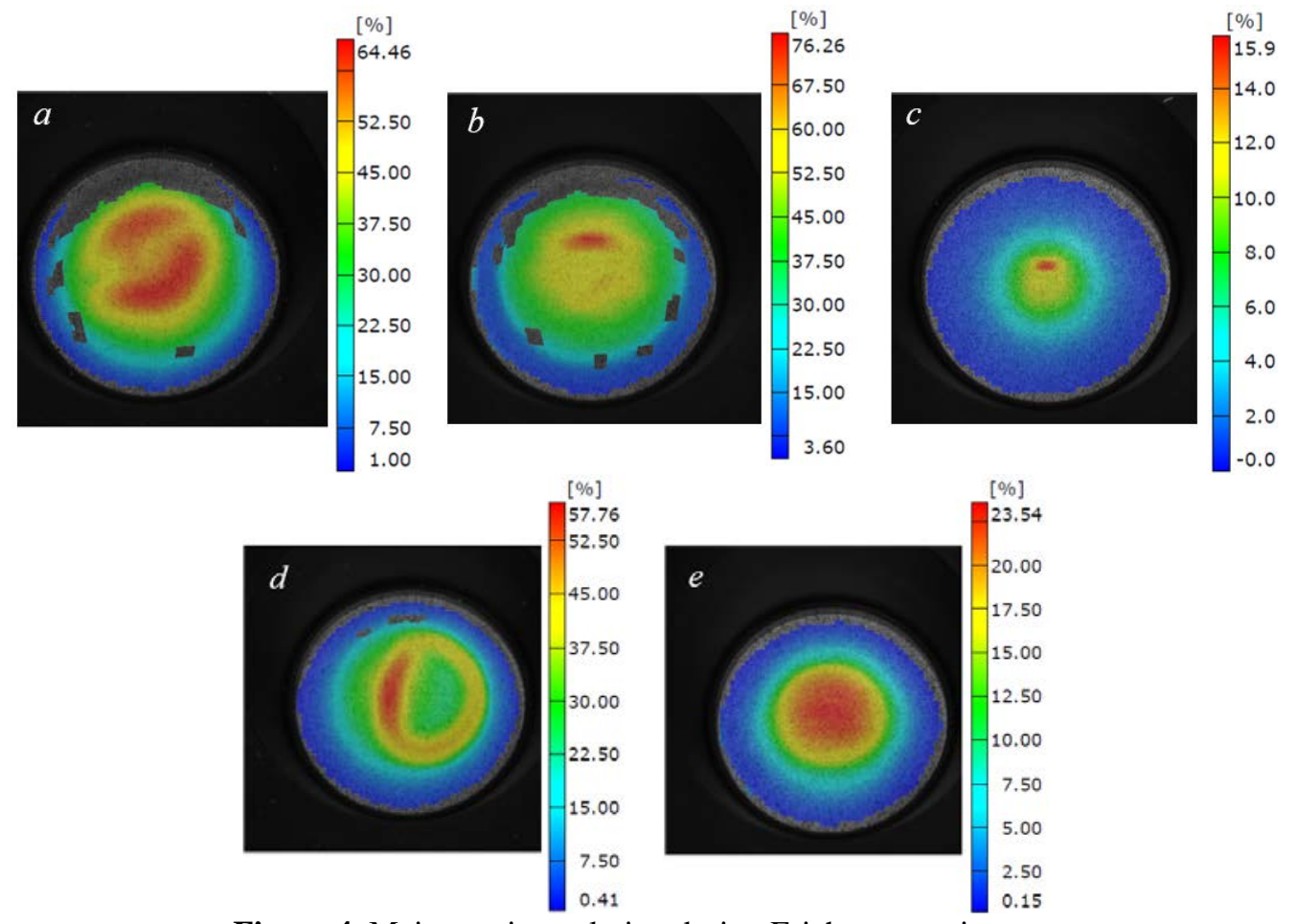

Figure 4. Major strain evolution during Erichsen cupping test (a)CP-Ti (Grade 2), (b)Ti-35A, (c)Ti-64, (d)Ti-215, (e)Ti-325
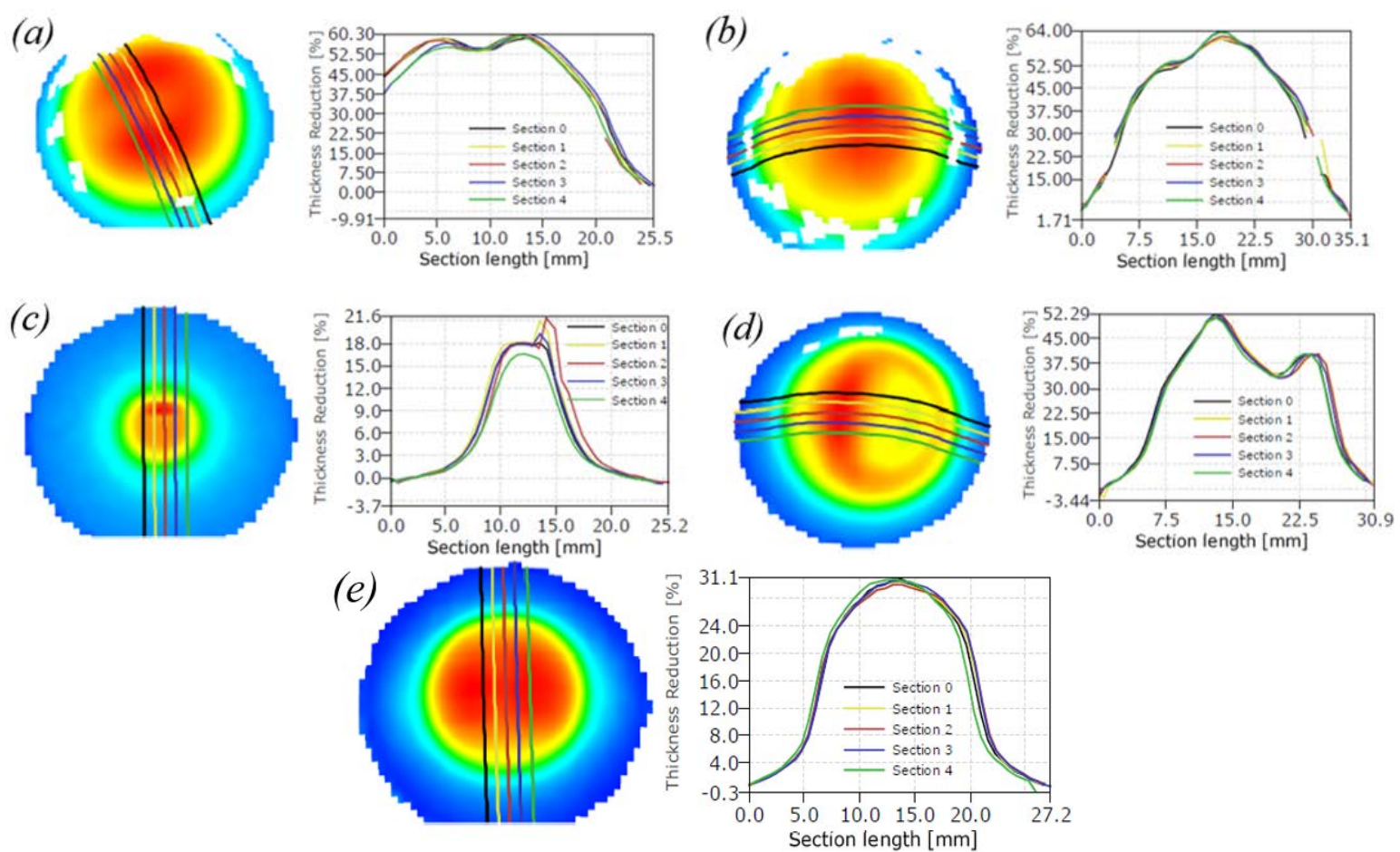

Figure 5. Thinning evolution during Erichsen test (a)CP-Ti (Grade 2), (b)Ti-35A, (c)Ti-64, (d)Ti-215, (e)Ti-325 


\subsection{Model predictability assessment and regression model equation}

Figure 6 shows the results of the generated regression equation coefficients and their respective statistical parameters, based on the experimental results run by the model fitting expression stated in equation (2) using $R$ programming.

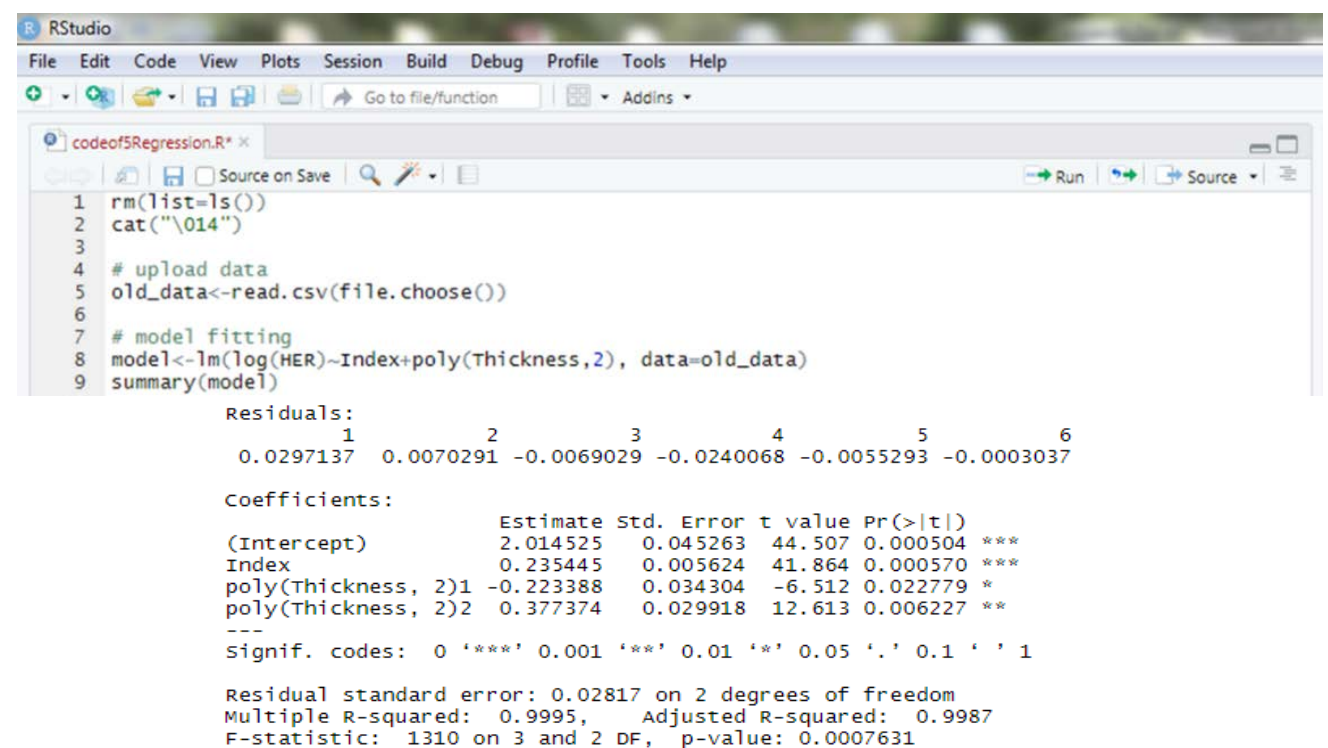

Figure 6. Generated regression model equation coefficients

Inspection of the $p$-values generated for the regression model equation coefficients revealed that all the coefficients have $p<0.05$. This shows that all the regression model equation coefficients respond well to the HER values. The overall regression model equation $p$-value (0.00076) also shows extreme statistical significance (see table 2), indicating the robustness of the model to predict the HER values of titanium alloys. $R^{2}{ }_{\text {Adj }}$ value of 0.9987 for the generated regression model equation also shows how well the regression line fits the data for accurate prediction of the HER values of titanium alloys. Therefore, for edges prepared with AWJ machining and deformed with a Nakajima punch, the proposed regression model equation for predicting HER of titanium alloys, based on the $E I$ and $t$ values is given by;

$$
H E R=e^{2.015+0.234 E I-0.223 t+0.377 t^{2}}
$$

Equation (11) generally takes the form of;

$$
H E R=e^{\beta_{0}+\beta_{1} E I+\beta_{2} t+\beta_{3} t^{2}}
$$

Where $\beta_{n}$ is regression coefficient, $n$ is an integer.

In order to validate the predictability of the model fitting expression, a cross-validation process was employed where the model was re-run with a deliberate omission of one experimental data point for all test values. For each validation round, the omitted experimental HER is compared to the predicted HER for each tested EI and $t$ value, generated from the remaining five-data model fitting by the expression in equation (2). A cross-validation process adopted for the onward prediction of the HER of Ti-6Al-4V using the R-script coded and executed in this research are shown in section 3.5. Similar R script executions were conducted for each titanium alloy examined in this research.

Table 4 shows the $p$-values obtained for the regression model equation coefficients of each titanium alloy used for predicting their HER values and those generated for the overall regression model equation as well as their corresponding $R^{2}{ }_{\text {Adj } j}$ values.

Generally, the obtained $p$-values suggests that the generated regression model equation coefficients respond well to the HER for all the titanium alloys examined. The overall $p$-values of the regression model equations generated also showed high statistical significance with their corresponding $R^{2}{ }_{A d j}$ values indicating a strong data fitting to the regression line, signifying the high statistical accuracy of the model. However, it is worth nothing that the robustness of the generated regression model equation coefficients are significantly improved, when all six data sets are fitted by the regression model expression. This shows that the model could be improved with 
further data sets, even though the statistical indicators are strong enough to predict HER values for titanium alloys in this work.

Table 4. $p$-value and $R^{2}$ ddj of generated regression model equation coefficients

\begin{tabular}{cllccll}
\hline Material & \multicolumn{1}{c}{$\boldsymbol{\beta}_{\mathbf{0}}$} & $\boldsymbol{\beta}_{\mathbf{1}}$ & $\boldsymbol{\beta}_{\mathbf{2}}$ & $\boldsymbol{\beta}_{\mathbf{3}}$ & Overall $\boldsymbol{p}$ & $\boldsymbol{R}^{\mathbf{2}}$ Adj \\
\hline $\mathrm{Ti}-325(1.6)$ & 0.00781 & 0.0078 & 0.04324 & 0.02699 & 0.01066 & 0.9997 \\
$\mathrm{Ti}-325$ & 0.022 & 0.0226 & 0.1208 & 0.0781 & 0.02908 & 0.9979 \\
$\mathrm{Ti}-215$ & 0.0205 & 0.0226 & 0.1106 & 0.0975 & 0.0277 & 0.9981 \\
$\mathrm{Ti}-64$ & 0.00381 & 0.00399 & 0.0164 & 0.01027 & 0.00474 & 0.9999 \\
$\mathrm{CP}-\mathrm{Ti}$ & 0.0354 & 0.0397 & 0.2492 & 0.0692 & 0.02877 & 0.988 \\
$\mathrm{Ti}-35 \mathrm{~A}$ & 0.0204 & 0.0226 & 0.1781 & 0.593 & 0.04186 & 0.9957 \\
All six & $\mathbf{0 . 0 0 0 5 0 4}$ & $\mathbf{0 . 0 0 0 5 7}$ & $\mathbf{0 . 0 2 2 7 7 9}$ & $\mathbf{0 . 0 0 6 2 2 7}$ & $\mathbf{0 . 0 0 0 7 6 3 1}$ & $\mathbf{0 . 9 9 8 7}$ \\
\hline
\end{tabular}

\subsection{Cross-validation process for predicting HER of Ti-6Al-4V}

Figure 7 shows the predicted HER of Ti-6Al-4V based on known EI $(4.0144 \mathrm{~mm})$ and t $(1.6 \mathrm{~mm})$ values. The generated regression model equation based on cross validation for the onward prediction of the HER value is given by equation (13) with an overall $p$-value of 0.0047 and $\mathrm{R}^{2}{ }_{\text {Adj }}=0.99$.

$$
H E R=e^{2.085+0.228 E I-0.234 t+0.393 t^{2}}
$$

(a)

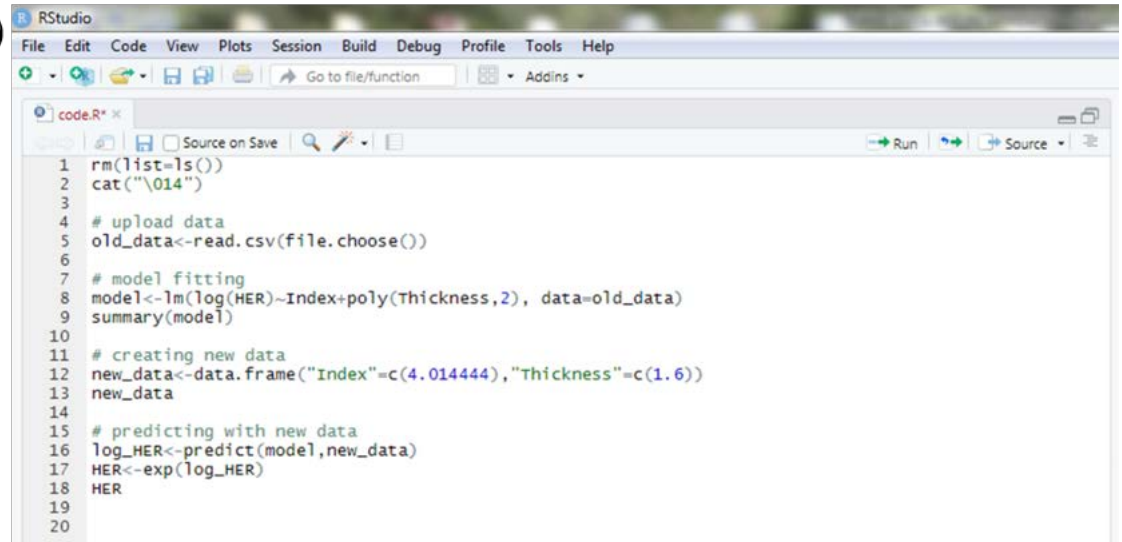

(b)

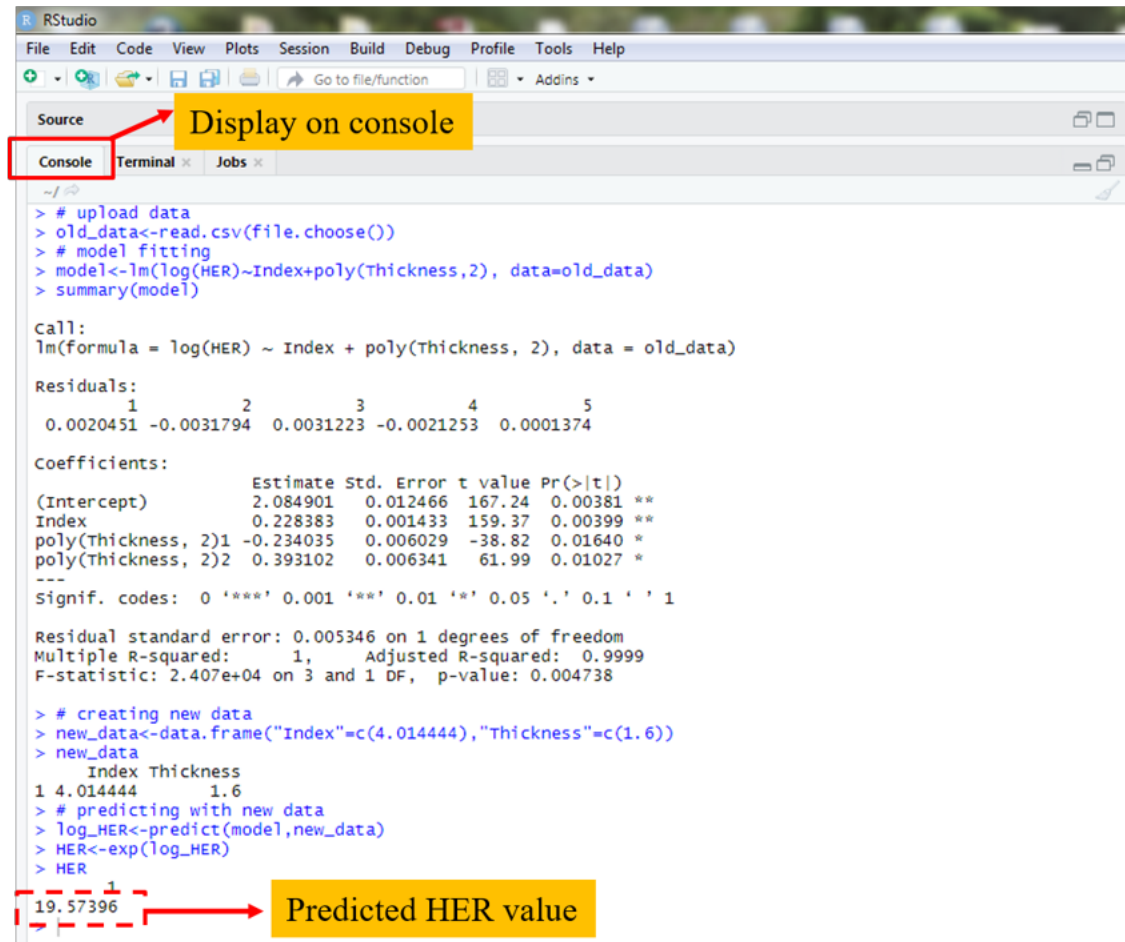

Figure 7. R-script computation on the user interface for Ti-6Al-4V.(a) Executed R-codes, (b) display on console 


\subsection{Comparison of the experimental and predicted HER}

Table 5 shows the experimental and the predicted HERs based on the proposed model fitting expression in equation (2). The results show very high proximity of the predicted HER values to the experimental HER values. This strength in proximity are consistent with the strong statistical indicators observed for the generated regression model equations, obtained for each validation trial. However, the predicted HER of Ti35A deviated significantly from the experimental HER, which was registered as high $p$-values in the $\boldsymbol{\beta}_{2}$ and $\boldsymbol{\beta}_{3}$ coefficients (table 4 ). The high $p$-values recorded represents a high drift in capturing variation in the data and a low correlation between factors. This could be attributed to the fact that Ti-35A was set as the optimum threshold for material performance in this work due to its superior ductility. Therefore utilising CP-Ti (Grade 2) as the predictor- model -calibrationthreshold value to capture Ti-35A was insufficient, because it has a lower HER value (81.9\%) compared to Ti35A (157.3\%). Thus, the omission of Ti-35A during the cross-validation trial produces a performance variability in their prediction response with the data set available, hence the deviation.

Table 5. Comparison of experimental and predicted HER

\begin{tabular}{cccc}
\hline Material & $\boldsymbol{t}, \mathbf{m m}$ & Experimental HER, \% & Predicted HER, \% \\
\hline Ti-3Al-2.5V & 1.60 & 30.096 & 28.726 \\
Ti-3Al-2.5V & 1.25 & 28.357 & 27.887 \\
Ti-2Al-1.5V & 1.27 & 52.529 & 53.4304 \\
Ti-6Al-4V & 1.60 & 18.344 & 19.574 \\
CP-Ti (Grade 2) & 1.60 & 81.870 & 84.647 \\
Ti-35A & 1.00 & 157.317 & 231.597 \\
\hline
\end{tabular}

A plot of the predicted and the experimental HERs of the examined titanium alloys is shown in Figure 8. The plot shows a strong linear relationship between the predicted and the experimental HERs. These results confirm the high robustness of the model fitting expression and the high predictability of the regression model equations generated.

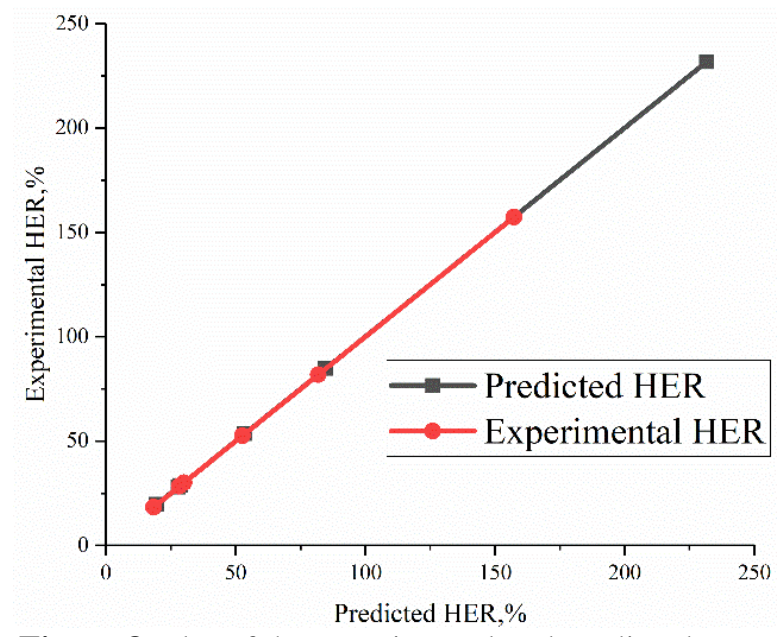

Figure 8. Plot of the experimental and predicted HER

Standard statistical constraint optimization parameter equations [28], are adopted to examine the results obtained in this work. The average absolute error $\left(\delta_{a b s}\right)$ obtained between the experimental HER (HER exp $)$ and the predicted HER (HER pre$_{\text {) }}$ is given by;

$$
\delta_{a b s}=\frac{1}{N} \sum_{i=1}^{N} \frac{H E R_{e x p}^{i}-H E R_{p r e}^{i}}{H E R_{e x p}^{i}}
$$

where $N$ is the number of data points considered.

Putting values from Table 5 into equation (14), the average absolute error obtained between the predicted and experimental HER in the studied titanium alloys, based on the proposed generated regression model equations is $8.8 \%$. 
In order to ascertain numerically, the strength of the linear relationship between the experimental and predicted HERs obtained from the generated regression model equations, the correlation coefficient $R$ is evaluated according to the equation;

$$
R=\frac{\sum_{i=1}^{N}\left(H E R_{\text {exp }}^{i}-\overline{H E R_{\text {exp }}}\right)\left(H E R_{\text {pre }}^{i}-\overline{H E R_{\text {pre }}}\right)}{\sqrt{\sum_{i=1}^{N}\left(H E R_{\text {exp }}^{i}-\overline{H E R_{\text {exp }}}\right)^{2} \sum_{i=1}^{N}\left(H E R_{\text {pre }}^{i}-\overline{H E R_{\text {pre }}}\right)^{2}}}
$$

where $\overline{H E R_{\text {exp }}}$ is the average experimental HER and $\overline{H E R_{\text {pre }}}$ is the average predicted HER

Putting values from Table 5 into equation (15), the correlation coefficient for the titanium alloys, based on the proposed generated regression model equations is given by;

$$
R=\frac{20800.95198}{\sqrt{442910563.4}}=0.9884
$$

The correlation coefficient of 0.9884 shows a strong linear relationship between the experimental and predicted HER values obtained for the titanium alloys. This result confirms the high robustness of the proposed regression model fitting expression for predicting the HER values in titanium and its alloys.

\section{Conclusions}

The stress state observed at the hole edge of sheet metals after HET are synonymous to those observed during conventional uniaxial tensile testing. However, conventional tensile test methods are not adequate in evaluating the edge forming performance of sheet metals. This similarity in stress state has led some researchers to correlate the tensile properties of sheet metals to their HERs. However, for highly anisotropic materials like titanium and its alloys, predicting the HER based solely on the tensile mechanical properties may not be representative of the material response. This work proposed a model fitting expression, which can be called in R programming language to predict the HER as well as generating regression model equations of titanium alloys based on known EI and $t$ values. The work found that;

1) The Erichsen cupping test based on biaxial stretching provides a route to assess the plastic formability of materials in all sheet-processing directions. This approach offers a better means of assessing the material forming performance as opposed to the limited routes offered by uniaxial tensile properties. The EI and HER offered similar trends of material formability thereby making a good case for correlating both parameters

2) Apart from the major role machining induced defects play in sheet edge formability, the material microstructure response also makes a significant impact on edge formability performance of titanium alloys

3) The HER of titanium alloys can be predicted based on known EI and $t$ values, when run in R programming with the model fitting expression:

$\operatorname{lm}(\log ($ HER $) \sim$ Erichsen Index + poly (thickness, 2), data = experimental data)

4) For hole edges prepared with AWJ and tested with a Nakajima punch, the HER of titanium alloys can be predicted, using the regression model equation:

$$
H E R=e^{2.015+0.234 E I-0.223 t+0.377 t^{2}}
$$

5) The proposed regression model equation has an overall $p$-value of 0.00076 indicating an extreme statistical significance and signifying the robustness of the model fitting expression to predict HER values of titanium alloys. $R^{2}$ Adj value of 0.9987 for the generated regression model equation also shows how well the regression line fits the data for accurate prediction of the HER of titanium alloys

6) A correlation coefficient of 0.9884 was obtained between the experimental and predicted HER values showing a strong linear relationship and a high degree of fit with an average absolute error of $8.8 \%$

\section{Acknowledgement}

The authors acknowledge the support of the Advanced Forming Research Centre (AFRC) and the Department of Design, Manufacturing and Engineering Management (DMEM) of the University of Strathclyde, Glasgow, UK. The authors are also thankful to TIMET for material supply. 


\section{References}

[1] Tsoupis I, Merklein M. Edge crack sensitivity of lightweight materials under different load conditions. IOP Conference Series: Materials science and Engineering. 2016; 159:1-8.

[2] Zhang Y, Liu Y, Wu X. Fracture behaviour of a dual phase steel in hole expansion test. 2007 ASME International Mechanical Engineering Congress and Exposition. Washington. 2009. p. 37-38

[3] Butcher C, Anderson D, Worswick M. Predicting failure during sheared edge stretching using a damagebased model for the shear-affected zone. SAE International Journal of Materials and Manufacturing. 2013;6(2):304-312.

[4] Park S, Jung J, Kim S, Seo SJ, Lee MG, Han HN. Prediction of fracture behavior in hole expansion test using microstructure based dual-scale model. Journal of Physics: Conference Series. 2018;1063:1-6.

[5] Chung K, Ma N, Park T, Kim D, Yoo D, Kim C. A modified damage model for advanced high strength steel sheets. International Journal of Plasticity. 2011;27(10):1485-1511.

[6] Paul SK, Mukherjee M, Kundu S, Chandra S. Prediction of hole expansion ratio for automotive grade steels. Computational Materials Science. 2014;89:189-197.

[7] Kumar De S, Deva A, Mukhopadhyay S, Kumar Jha B. Assessment of formability of hot-rolled steel through determination of hole-expansion ratio. Materials and Manufacturing Processes. 2011;26(1):37-42.

[8] Chen X, Jiang H, Cui Z, Lian C, Lu C. Hole expansion characteristics of ultra high strength steels. Procedia Engineering. 2014;81:718 -723.

[9] Fang X, Fan Z, Ralph B, Evans P, Underhill R. The relationships between tensile properties and hole expansion property of C-Mn steels. Journal of Materials Science. 2003;38:3877-3882.

[10] Adamczyk RD, Michal GM. Sheared edge extension of high-strength cold-rolled steels. Journal of Applied Metal Working. 1986;4(2):157-163.

[11] Kim JH, Kwon YJ, Lee T, Lee KA, Kim HS, Lee CS. Prediction of hole expansion ratio for various steel sheets based on uniaxial tensile properties. Metals and Materials International. 2018;24(1):187-194.

[12] Pathak N, Butcher C, Worswick M. Assessment of the critical parameters influencing the edge stretchability of advanced high-strength steel sheet. Journal of Materials Engineering and Performance. 2016;25(11):49194932.

[13] Singh AK, Schwarzer RA. Texture and anisotropy of mechanical properties in titanium and its alloys. Zeitschrift für Metallkunde. 2000;91(9):702-716.

[14] Kocańda A, Jasiński C. Extended evaluation of Erichsen cupping test results by means of laser speckle. Archives of Civil and Mechanical Engineering. 2016;16(2):211-216.

[15] Aydin M, Wu X, Cetinkaya K, Yasar M, Kadi I. Application of digital image correlation technique to Erichsen cupping test. Engineering Science and Technology, An International Journal. 2018;21(4):760-768.

[16] Santos RO, Pereira AB, Butuc MC, Vincze G, Festas AJ, Moreira LP. Development of a device compatible with universal testing machine to perform hole expansion and Erichsen cupping tests. Machines. 2020;8(1):18.

[17] Dilmec M, Halkaci HS, Ozturk F, Livatyali H, Yigit O. Effects of sheet thickness and anisotropy on forming limit curves of AA2024-T4. The International Journal of Advanced Manufacturing Technology. 2013;67:2689-2700.

[18] Zadpoor AA, Sinke J, Benedictus R. The Effects of thickness on the formability of 2000 and 7000 series high strength aluminum alloys. Key Engineering Materials. 2009;410-411:459-466.

[19] Hashemi A, Mashhadi M, Bakhshi-Jooybari M, Gorji A. Study of the effect of material properties and sheet thickness on formability of conical parts in hydro-mechanical deep drawing assisted by radial pressure. Advanced Materials Research. 2012;445:149-154.

[20] Beal JD, Boyer R, Sanders D. Forming of titanium and titanium alloys. In: Semiatin SL ed. Metalworking: Sheet Forming. ASM Handbook. 2006;14B:656-669.

[21] Jones O, Maillardet R, Robinson A. Introduction to scientific programming and simulation using R. Second ed. Boca Raton: CRC Press, Taylor \& Francis Group. 2014.

[22] Verzani J. Using R for introductory statistics. Second ed. New York: CRC Press, Taylor \& Francis Group. 2014.

[23] Bloomfield VA. Using R for numerical analysis in science and engineering. First ed. Boca Raton: CRC Press, Taylor \& Francis Group. 2014.

[24] Harel O. The estimation of $\mathrm{R}^{2}$ and adjusted $\mathrm{R}^{2}$ in incomplete data sets using multiple imputation. Journal of Applied Statistics. 2009;36(10):1109-1118.

[25] Badr OM, Rolfe B, Hodgson P, Weiss M. Forming of high strength titanium sheet at room temperature. Materials and Design. 2015;66(Part B):618-626. 
[26] Lin Prakash PS, Rajak B, Panda SK, Roy GG, Jha MN, Mascarenhas M. Mechanical properties and stretch forming behaviour of electron beam welded titanium sheets of grade-2 and grade-5. Materials Today: Proceedings. 2017;4(2 Part A):908-916.

[27] Kurra S, Regalla SP. Experimental and numerical studies on formability of extra-deep drawing steel in incremental sheet metal forming. Journal of Materials Research and Technology. 2014;3(2):158-171.

[28] Kotkunde N, Krishnamurthy HN, Singh SK, Jella G. Experimental and numerical investigations on hot deformation behavior and processing maps for ASS 304 and ASS 316. High Temperature Materials and Processes. 2018;37(9-10):873-888.

(C) 2020 by the author(s). This work is licensed under a Creative Commons Attribution 4.0 International License (http://creativecommons.org/licenses/by/4.0/). Authors retain copyright of their work, with first publication rights granted to Tech Reviews Ltd. 